\title{
Effect of thermal annealing on the metastable optical properties of GaN thin films
}

\author{
Y. C. Chang ${ }^{a)}$ \\ Institute of Electro-Optical Science and Engineering and Advanced Optoelectric Technology Center, \\ National Cheng Kung University, Tainan 701, Taiwan, Republic of China \\ R. M. Kolbas \\ Department of Electrical and Computer Engineering, North Carolina State University, \\ Raleigh, North Carolina 27695-7911 \\ Z. J. Reitmeier and R. F. Davis \\ Department of Materials Science and Engineering, North Carolina State University, \\ Raleigh, North Carolina 27695-7907
}

(Received 3 March 2006; accepted 8 May 2006; published 12 June 2006)

\begin{abstract}
The optical metastability in unintentionally doped GaN (0001) films grown on AlN/SiC substrates has been investigated as a function of thermal annealing conditions using photoluminescence and optical microscopy. Annealing at $800{ }^{\circ} \mathrm{C}$ for $48 \mathrm{~h}$ in 1 atm of flowing nitrogen produced no change in the metastability. Annealing at $800{ }^{\circ} \mathrm{C}$ in ultrahigh vacuum for $48 \mathrm{~h}$ eliminated the phenomenon. Exposure of the sample to ultraviolet light during the latter anneal reduced the time to eliminate the metastability. This phenomenon was restored by subsequently annealing in ammonia at $775^{\circ} \mathrm{C}$ for $3 \mathrm{~h}$. These results suggest that the presence and elimination of the optical metastability are related to the presence in and the elimination of hydrogen from the GaN. (C) 2006 American Vacuum Society. [DOI: 10.1116/1.2209656]
\end{abstract}

The III-nitrides are the leading candidate materials for next generation high-power, ultraviolet emitting optoelectronic devices due to their strong interatomic bonds and large direct band-gap energies. GaN is the principal paradigm and the most studied of this group of materials. The progress in material growth of thin films and thin boules of these nitrides has been significant. ${ }^{1,2}$ The origin of the most common, stable, parasitic, defect-induced emission centered around $2.3 \mathrm{eV}$ in unintentionally doped $\mathrm{GaN}$, commonly referred to as yellow luminescence (YL), is still not well understood. Recent results from first-principles calculations ${ }^{3}$ and positron annihilation experiments ${ }^{4}$ indicate that this luminescence is produced by transitions between a shallow donor level and a deep acceptor level introduced by gallium vacancies.

Metastable effects in a semiconductor can influence the performance of a device. Electrical metastability in the form of persistent photoconductivity has been observed in silicondoped GaN thin films. ${ }^{5,6}$ Metastability in the photoluminescence spectra of undoped GaN, induced using ultraviolet (UV) light, has been reported by two other groups. ${ }^{7,8}$ Exposure to intense UV laser radiation was observed ${ }^{9,10}$ to produce metastable photoluminescence images in $\mathrm{InGaN} / \mathrm{GaN}$ heterostructures and multiple quantum well samples grown on sapphire substrates. Similar metastability was reported for undoped $\mathrm{GaN}$ grown on $\mathrm{SiC}$ substrates. ${ }^{11}$ Exposure to a $5 \mathrm{keV}$ electron beam also produced similar metastability. ${ }^{12}$ Either the elimination or the erasure of this metastability was enhanced at elevated temperatures; the thermal activation energy was $1.1 \mathrm{eV} .^{11}$ A second deep level emission centered

a) Author to whom correspondence should be addressed; electronic mail: ychang6@mail.ncku.edu.tw around $2.9 \mathrm{eV}$ was observed in all of the unintentionally doped GaN samples that exhibited the metastability. ${ }^{11,12}$ This deep level emission is referred to as the blue luminescence (BL). The origin of this emission has not been determined. Several theoretical calculations have predicted that hydrogenated gallium vacancies will induce deep acceptor levels within the band gap of GaN. ${ }^{13,14}$ Transition energies to these levels will be close to the energy of the BL. These studies suggest that hydrogen-related defect complexes in GaN may be the origin of both the BL and the metastability.

In this research, the existence of the optical metastability in unintentionally doped $\mathrm{GaN}$ films has been studied as a function of various annealing processes. Line patterns produced by intense ultraviolet laser illumination were observed on the samples that exhibited the optical metastability. This metastability was considered to be eliminated if the same laser illumination did not reproduce the patterns. Detailed information of the optical metastability were discussed in Ref. 11. Ultrahigh vacuum annealing eliminated the optical metastability, and annealing under ultraviolet illumination accelerated the removal processes. The BL intensity was greatly reduced in the subsequent PL spectra of the samples that no longer exhibited the optical metastability. Subsequent annealing in $\mathrm{NH}_{3}$ restored the metastability. These results suggest that the optical metastability and the BL are closely related to the hydrogen-related defects.

The samples used in this study were unintentionally doped GaN thin films deposited on AlN/6H-SiC(0001) substrates using a vertical, resistively heated, cold walled quartz metal organic chemical vapor deposition (MOCVD) reactor. The beam of a frequency tripled output of a mode locked Ti:sapphire laser (250 fs at $280 \mathrm{~nm}, 76 \mathrm{MHz}$ repetition rate) 
TABLE I. Relationships between thermal annealing conditions and optical metastability. Samples A, C, D, and F are all from the sample $1 \mu \mathrm{m}$ thick GaN thin film. Samples B and E are from a second, $0.3 \mu \mathrm{m}$ thick, GaN film grown under similar conditions.

\begin{tabular}{ccc}
\hline \hline Sample & $\begin{array}{c}\text { Annealing } \\
\text { condition }\end{array}$ & $\begin{array}{c}\text { Memory } \\
\text { effect? }\end{array}$ \\
\hline A & Before annealing & Yes \\
B & $800{ }^{\circ} \mathrm{C} / 48 \mathrm{~h} /$ flowing $\mathrm{N}_{2}$ & Yes \\
$\mathrm{C}$ & $800{ }^{\circ} \mathrm{C} / 48 \mathrm{~h} / \mathrm{UHV}$ & No \\
$\mathrm{D}$ & $800{ }^{\circ} \mathrm{C} / 48 \mathrm{~h} / \mathrm{UHV} /+775^{\circ} \mathrm{C} / 3 \mathrm{~h} / \mathrm{NH}_{3}$ & Yes \\
$\mathrm{E}$ & $800{ }^{\circ} \mathrm{C} / 3 \mathrm{~h} / \mathrm{UHV} / \mathrm{UV}$ laser & No \\
$\mathrm{F}$ & $800^{\circ} \mathrm{C} / 3 \mathrm{~h} / \mathrm{UHV}$ & Yes \\
\hline \hline
\end{tabular}

was focused to a spot size of $\sim 20 \mu \mathrm{m}$ and translated across selected samples to produce metastable patterns that could be viewed under low intensity UV illumination through an optical microscope. These patterns gradually disappeared with time and new patterns could be produced on the same area of the sample. Detailed information regarding the conditions used to grow the films and the read and write procedures employed to produce the metastable patterns are reported in Ref. 11.

The GaN samples were annealed in a high purity flowing nitrogen atmosphere in a quartz-walled furnace. The ultrahigh vacuum (UHV) annealing was performed with a resistive heater located inside the vacuum chamber. The sapphire viewpoint of this vacuum chamber allowed ultraviolet laser illumination on the sample while annealing. Annealing in $\mathrm{NH}_{3}$ was performed in the same MOCVD chamber used to grow the samples.

Photoluminescence (PL) measurements were performed using the same UV laser mentioned above as the excitation source, and the signals were analyzed by a $0.3 \mathrm{~m}$ spectrometer and a cooled GaAs photomultiplier. The room temperature PL spectra acquired at 100 and $20 \mathrm{~W} / \mathrm{cm}^{2}$ from one of the sections cut from the unannealed GaN film (sample A in Table I) that exhibited optical metastability are shown in Fig. 1. Narrow [full width at half maximum $(\mathrm{FWHM})=30 \mathrm{meV}$ ] band-to-band emission at $3.4 \mathrm{eV}$ was observed in the spectra, indicating that the GaN thin films do not contain significant

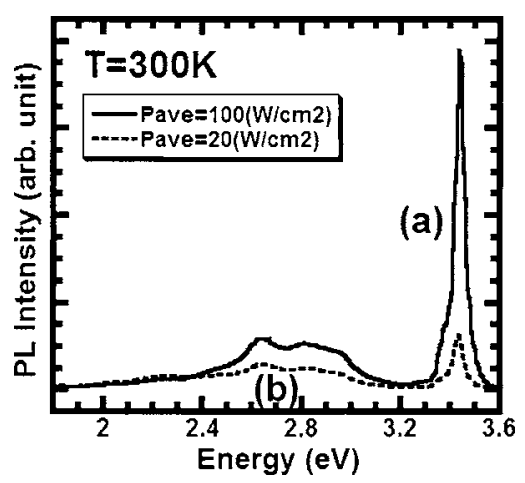

FIG. 1. Room temperature photoluminescence spectra acquired at $300 \mathrm{~K}$ using excitation power densities of (a) 100 and (b) $20 \mathrm{~W} / \mathrm{cm}^{2}$ from an unannealed GaN sample (sample A) that exhibited metastability.

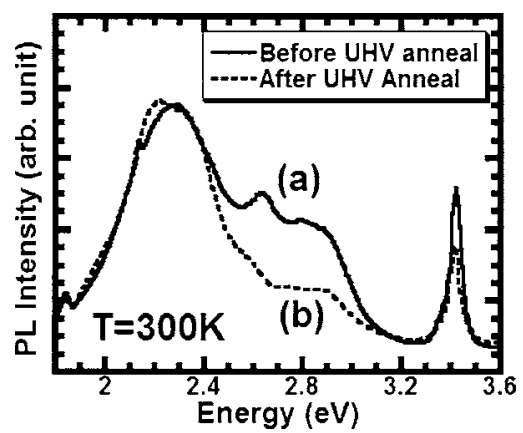

FIG. 2. Room temperature photoluminescence spectra acquired at $300 \mathrm{~K}$ using an excitation power density of $1.6 \mathrm{~W} / \mathrm{cm}^{2}$ from an unannealed $\mathrm{GaN}$ sample [spectrum (a)] and a similar sample annealed in an ultrahigh vacuum $\left(\sim 10^{-9}\right.$ Torr $)$ at $800^{\circ} \mathrm{C}$ for $48 \mathrm{~h}$ [spectrum (b)].

structural or microstructural damage in addition to the high density of threading dislocations present in essentially all heterostructural GaN films. Deep level emission peaks centered around $2.2(\mathrm{YL})$ and $2.9 \mathrm{eV}(\mathrm{BL})$ were also observed. The BL emission always appeared in the PL of the as-grown samples that exhibited optical metastability.

Deep level spectra in semiconductor materials can usually be explained by the presence of either point defects including unintentionally added impurities and vacancies or one- and two-dimensional defects including dislocations, stacking faults, and antiphase boundaries. If the spectra are produced by the excitation of impurities, thermal annealing and the associated breaking of chemical bonds and the migration of these defects should provide a mechanism to either alter the crystallographic site of or eliminate the species from the sample and thereby change or reduce the spectra from these defects and provide some understanding of their properties. As such, thermal annealing under different conditions was performed on samples that exhibited metastability. The impact of the different annealing conditions on this metastability is summarized in Table I. One of the as-grown samples was annealed in flowing nitrogen at $800{ }^{\circ} \mathrm{C}$ for $48 \mathrm{~h}$ (sample B). No changes in the PL spectra were observed within this period and this sample still exhibited optical metastability in the laser-written pattern after the annealing.

Annealing another unannealed sample in an ultrahigh vacuum $\left(\sim 10^{-9}\right.$ Torr) at $800{ }^{\circ} \mathrm{C}$ for $48 \mathrm{~h}$ (sample $\mathrm{C}$ ) did not cause visible damage to the surface of the film, as observed using several magnifications in an optical microscope. However, the annealed sample no longer exhibited the optical metastability in the written pattern. Room temperature PL measurements performed before and after this UHV anneal using the excitation power density of $1.6 \mathrm{~W} / \mathrm{cm}^{2}$ are shown in Fig. 2. A decrease in the intensity of the BL was observed in the UHV-annealed sample; however, the intensity of YL remained roughly unchanged. This suggests that the BL and the observed optical metastability are linked, especially in light of the PL results observed after the $\mathrm{NH}_{3}$ annealing studies described below.

The laser used to acquire the spectra shown in Fig. 2 was used to illuminate part of a previously unannealed sample 


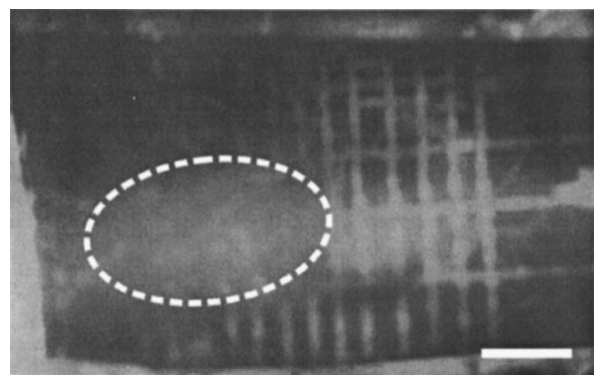

FIG. 3. Room temperature low level PL image of sample E taken through the eye piece of a microscope after annealing. (Reference marker is $1 \mathrm{~mm}$.) The area within the oval of the sample was illuminated using an ultraviolet laser with an excitation power density of $1.6 \mathrm{~W} / \mathrm{cm}^{2}$ during heating of the entire sample at $800{ }^{\circ} \mathrm{C}$ in UHV for $3 \mathrm{~h}$. The lighter vertical and horizontal lines are metastable patterns that were subsequently written across the sample. The UV illuminated area of the sample does not exhibit optical metastability and no patterns can be produced within the oval of the sample. The remainder of the sample still exhibits optical metastability and line patterns can be written and observed.

during a second $\mathrm{UHV}$ anneal at $800{ }^{\circ} \mathrm{C}$ for $3 \mathrm{~h}$ (sample E). Subsequently, vertical and horizontal optical metastability lines were written across the sample. The room temperature PL image under low intensity UV illumination acquired from this sample after the annealing is shown in Fig. 3. The oval indicates the laser-illuminated area during the annealing. The same intense laser light that can produce metastability lines (outside the oval) cannot produce metastability patterns within the laser-illuminated area (inside the oval). The results indicate that annealing in UHV for $3 \mathrm{~h}$ without the UV illumination cannot eliminate the optical metastability. The addition of UV illumination significantly enhanced the elimination of the optical metastability. This result suggests that the ultraviolet light either enhances the thermal annealing process or generates a second mechanism to eliminate the cause of the optical metastability.

Results from the UHV annealing study also indicate that a long period of thermal annealing in UHV alters the sample's properties. Hydrogen has been reported to have low thermal diffusivity in $n$-type GaN. ${ }^{15}$ Optical transitions to the levels induced by hydrogenated gallium vacancies may explain the BL spectra. We suggest that annealing of the GaN in UHV at $800{ }^{\circ} \mathrm{C}$ for $48 \mathrm{~h}$ causes the diffusion of hydrogen atoms inside the material and the desorption of molecular $\mathrm{H}_{2}$ from the surface of the film that ultimately results in a decrease in the BL spectra. To support this hypothesis, the UHV-annealed sample $\left(800{ }^{\circ} \mathrm{C} / 48 \mathrm{~h} / \mathrm{UHV}\right)$ was subsequently annealed at $775{ }^{\circ} \mathrm{C}$ in the original growth chamber in flowing $\mathrm{NH}_{3}$ for $3 \mathrm{~h}$ (sample D). This sample and the sample annealed in UHV at $800{ }^{\circ} \mathrm{C}$ for $48 \mathrm{~h}$ (sample C) were subsequently investigated for optical metastability by producing line patterns on the area within the oval of Fig. 4 under the same excitation power density. This intense laser light cannot produce metastability lines on the UHV-annealed sample (sample C), but can produce metastability patterns on the subsequently $\mathrm{NH}_{3}$-annealed sample (sample D). The results indicate that annealing in $\mathrm{NH}_{3}$ for $3 \mathrm{~h}$ reverses the elimination of optical metastability. At this stage only sample D exhibited the op-

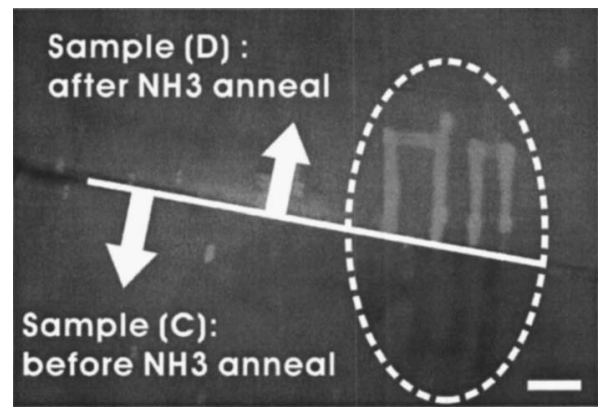

FIG. 4. Room temperature images of samples C and D observed through the eye piece of an optical microscope when they were illuminated using an ultraviolet laser with an excitation power density of $1.6 \mathrm{~W} / \mathrm{cm}^{2}$. (Reference marker is $0.2 \mathrm{~mm}$.) Both samples had been previously annealed in UHV at $800{ }^{\circ} \mathrm{C}$ for $48 \mathrm{~h}$ and did not exhibit optical memory effects. Sample D was subsequently annealed in $\mathrm{NH}_{3}$. The line patterns can be produced in sample $\mathrm{D}$, but not in sample $\mathrm{C}$. (See the pattern inside the oval.) The optical metastability is restored by the subsequent anneal in $\mathrm{NH}_{3}$.

tical metastability. This result is in agreement with our hypothesis that the presence of hydrogen in the GaN films is closely related to the presence of the optical metastability observed in the samples.

Based on our results from various annealing processes, we believe that the observed optical metastability is related to hydrogen/defect complexes, probably hydrogenated gallium vacancies. A long-time anneal in UHV at high temperature is required to remove the hydrogen molecules from the sample and to eliminate this optical metastability. This is in agreement with the reported ${ }^{14}$ low diffusivity of hydrogen atoms in $n$-type GaN. An ultraviolet photon has sufficient energy $(>4.0 \mathrm{eV})$ to break the bonds between the hydrogen and the lattice atoms and enhance the hydrogen diffusion, which explains the results for the shorter annealing time (sample E). Hydrogen atoms can be reintroduced and the optical metastability restored by annealing in a hydrogenrich environment such as that provided by $\mathrm{NH}_{3}$.

In summary, thermal annealing of $\mathrm{GaN}(0001)$ thin films grown by MOCVD on AlN/SiC(0001) substrates was performed at (a) $800{ }^{\circ} \mathrm{C}$ in $1 \mathrm{~atm}$ of flowing nitrogen for $48 \mathrm{~h}$, (b) $800{ }^{\circ} \mathrm{C}$ in UHV for $48 \mathrm{~h}$ either with or without exposure to an ultraviolet light, and (c) $775{ }^{\circ} \mathrm{C}$ in 45 Torr of ammonia for $3 \mathrm{~h}$. The sample annealed in nitrogen continued to exhibit the optical metastability. A $48 \mathrm{~h}$ long UHV anneal eliminated the optical metastability. Exposure of the $\mathrm{GaN}$ to ultraviolet light during the latter anneal reduced the time necessary to eliminate these metastable effects to $1 \mathrm{~h}$. The BL intensity in the photoluminescence spectra was reduced after the latter anneal. The optical metastability was restored during a subsequent anneal in ammonia. These results indicate that the optical metastability and the blue luminescence observed in the undoped GaN are diminished as the concentration of hydrogen in the crystal lattice is reduced. It is suggested that hydrogenated gallium vacancies are the origin of both the blue luminescence and the optical metastability observed in this study.

${ }^{1}$ R. F. Davis, A. M. Roskowski, E. A. Preble, J. S. Speck, B. Heying, J. A. 
Freitas, E. R. Glaser, and W. E. Carlos, Proc. IEEE 90, 993 (2002).

${ }^{2}$ S. Keller and S. P. DenBaars, J. Cryst. Growth 248, 479 (2003).

${ }^{3}$ J. Neugebauer and C. G. Van de Walle, Appl. Phys. Lett. 69, 503 (1996).

${ }^{4}$ K. Saarinen et al., Phys. Rev. Lett. 79, 3030 (1997).

${ }^{5}$ G. Beadie, W. S. Rabinovich, A. E. Wickenden, D. D. Koleske, S. C. Binari, and J. A. Freitas, Jr., Appl. Phys. Lett. 71, 1092 (1997).

${ }^{6}$ H. M. Chen, Y. F. Chen, M. C. Lee, and M. S. Feng, J. Appl. Phys. 82, 899 (1997).

${ }^{7}$ S. J. Xu, G. Li, S. J. Chua, X. C. Wang, and W. Wang, Appl. Phys. Lett. 72, 2451(1998).

${ }^{8}$ B. Kim, I. Kuskovsky, I. P. Herman, D. Li, and G. F. Neumark, J. Appl.
Phys. 86, 2034 (1999).

${ }^{9}$ I. K. Shmagin et al., Appl. Phys. Lett. 71, 1382 (1997).

${ }^{10}$ I. K. Shmagin et al., Appl. Phys. Lett. 71, 1455 (1997).

${ }^{11}$ Y. C. Chang, A. E. Oberhofer, J. F. Muth, R. M. Kolbas, and R. F. Davis, Appl. Phys. Lett. 79, 281 (2001).

${ }^{12}$ Y. C. Chang, A. L. Cai, M. A. L. Johnson, J. F. Muth, R. M. Kolbas, Z. J. Reitmeier, S. Einfeldt, and R. F. Davis, Appl. Phys. Lett. 80, 2675 (2002).

${ }^{13}$ C. G. Van de Walle, Phys. Rev. B 56, R10020 (1997).

${ }^{14}$ A. F. Wright, J. Appl. Phys. 90, 1164 (2001).

${ }^{15}$ A. Y. Polyakov et al., Appl. Phys. Lett. 79, 1834 (2001). 